\title{
Database of the PONDSCAPE project (Towards a sustainable management of pond diversity at the landscape level)
}

Pieter Lemmens (1D, Aaike De Wever, Nicolas Bonjean, Annick Castiaux, Liesbeth Colson, Tom De Bie, Evelyn Decoster, Carla Denis, Els De Roeck, Dirk Ercken, Boudewijn Goddeeris, Raphaëlla Govvaerts. Svaahalirwa N.M. Mandiki, Kevin Morelle, Emilie Praca,
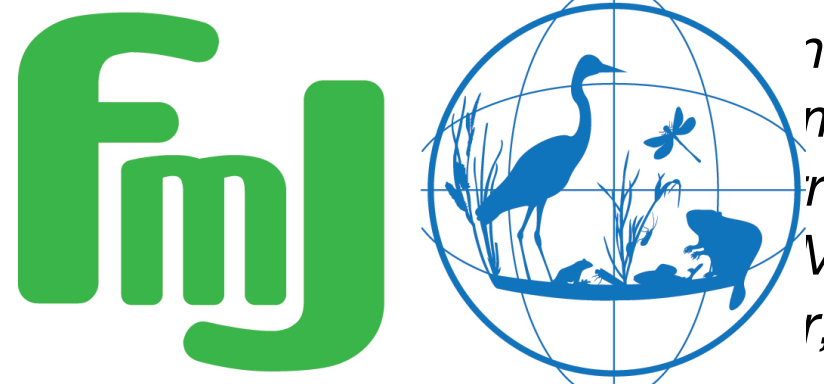

7, Katleen van der Gucht, Jochen melingen, Maria Villena Alvarez, Dirk remans, Leo Vanhecke, Betrand Vichel Cauchie, Patrick Kestemont, r, Steven A.J. Declerck \& Koen

Freshwater Metadata Journal 



\section{Database of the PONDSCAPE project (Towards a sustainable management of pond diversity at the landscape level)}

Pieter Lemmens ${ }^{1}$ (iD), Aaike De Wever ${ }^{2}$, Nicolas Bonjean ${ }^{5}$, Annick Castiaux ${ }^{4}$, Liesbeth Colson ${ }^{2}$, Tom De Bie ${ }^{1}$, Evelyn Decoster ${ }^{1}$, Carla Denis ${ }^{1}$, Els De Roeck ${ }^{2}$, Dirk Ercken ${ }^{2}$, Boudewijn Goddeeris ${ }^{2}$, Raphaëlla Goyvaerts ${ }^{1}$, Syaghalirwa N.M. Mandiki ${ }^{4}$, Kevin Morelle ${ }^{4}$, Emilie Praca ${ }^{5}$, Isa Schön ${ }^{2}$, Jeroen Van Wichelen ${ }^{3}$, Katleen van der Gucht ${ }^{3}$, Jochen Vandekerckhoven ${ }^{2}$, Pieter Vanormelingen ${ }^{3}$, Maria Villena Alvarez ${ }^{3}$, Dirk Bauwens ${ }^{6}$, Luc Denys $^{6}$, Marc Herremans ${ }^{8}$, Leo Vanhecke ${ }^{7}$, Betrand Losson ${ }^{9}$, Yannick Caron ${ }^{9}$, Henry-Michel Cauchie ${ }^{5}$, Patrick Kestemont ${ }^{4}$, Wim Vyverman ${ }^{2}$, Luc De Meester ${ }^{1}$, Steven A.J. Declerck ${ }^{1,10}$ \& Koen Martens ${ }^{2}$

$1 \mathrm{KU}$ Leuven, Laboratory of Aquatic Ecology, Evolution and Conservation, Leuven, Belgium; corresponding author: pieter.lemmens@kuleuven.be

2 Royal Belgian Institute of Natural Sciences, Freshwater Biology, Brussels, Belgium

3 Ghent University, Department of Biology, Ghent, Belgium

4 Université de Namur, Namur, Belgium

5 Centre de Recherche Public - Gabriel Lippmann (CRP-GL), Belvaux, Luxembourg

6 Research Institute for Nature and Forest, Brussels, Belgium

7 National Botanic Garden of Belgium, Meise, Belgium

8 Natuurpunt, Mechelen, Belgium

9 University of Liège, Department of Parasitology and Parasitic Diseases, Liège, Belgium

10 Present address: Netherlands Institute of Ecology, Department of Aquatic Ecology, Wageningen, The Netherlands

Please cite this paper as follows: Lemmens, P., De Wever, A., Bonjean, N., Castiaux, A., Colson, L., De Bie, T., Decoster, E., Denis, C., De Roeck, E., Ercken, D., Goddeeris, B., Goyvaerts, R., Mandiki, S.N.M., Morelle, K., Praca, E., Schön, I., Van Wichelen, J., van der Gucht, K., Vandekerckhoven, J., Vanormelingen, P., Villena Alvarez, M., Bauwens, D., Denys, L., Herremans, M., Vanhecke, L., Losson, B., Caron, Y., Cauchie, H.-M., Kestemont, P., Vyverman, W., De Meester, L., Declerck, S.A.J. \& Martens, K., 2018. Database of the PONDSCAPE project (Towards a sustainable management of pond diversity at the landscape level).

Freshwater Metadata Journal 31: 1-10. https://doi.org/10.15504/fmj.2018.31

Received: 2018-09-24 / Published: 2018-09-27

\section{Keywords}

farmland ponds, environmental conditions, occurrences, Belgium, Luxembourg

\section{Short description of the dataset/summary}

The database of the PONDSCAPE project (Towards a sustainable management of pond diversity at the landscape level) comprises taxon occurrence data of eight different organism groups (bacteria, phytoplankton, diatoms, cladoceran, macro-invertebrates, macrophytes, amphibians and fish) and data on physical, chemical and morphometric variables of 125 farmland ponds covering five biogeographic regions in Belgium and Luxembourg. 


\section{General information}

dataset entry ID:

name of the dataset:

full name of the dataset:

dataset short name:

type of dataset:

data type:

science keyw

\section{SF_9}

Towards a sustainable management of pond diversity at the landscape level Pondscape

species (taxonomic group) per site database including environmental information

point data/observation data topic:

ISO topic category according to ISO 19115:

Agriculture, Biosphere, Biological Classification, Land Surface, Terrestrial Hydrosphere

Farming, Biota, Environment, Inland Waters

INSPIRE keywords according to GEMET:

own science keywords:

related project:

funding:
Bio-geographical regions

farmland ponds, occurrences, environmental conditions, Belgium, Luxembourg PONDSCAPE

Belspo research project $\mathrm{SD} / \mathrm{BD} / 02 \mathrm{~A}$

\section{Technical and administrative specifications}

$\begin{array}{ll}\text { data format: } & \text { Excel } \\ \text { operating system: } & \text { all Windows systems } \\ \text { data language: } & \text { English } \\ \text { current access level: } & \text { internal } \\ \quad \text { currently available through GBIF: } & \text { yes } \\ \text { exchange planned: } & \text { yes } \\ \text { data in data repository: } & \text { no }\end{array}$

Do you plan to publish the data on the Freshwater Biodiversity Data Portal:

$\begin{array}{ll}\begin{array}{l}\text { update level: } \\ \text { documentation: } \\ \text { type: }\end{array} & \text { completed } \\ \text { language: } & \text { scientific paper } \\ & \text { English } \\ \text { contact details: } & \\ \text { metadata contact person: } & \\ \text { first, last name: } & \text { Koen Martens } \\ \text { phone: } & +32(0) 26274315 \\ \text { email: } & \text { koen.martens@naturalsciences.be } \\ \text { institution: } & \text { Royal Belgian Institute of Natural Sciences } \\ \text { address: } & \text { Vautierstraat } 29 \\ \text { postal code, city: } & 1000 \text { Brussels } \\ \text { country } & \text { Belgium } \\ \text { technical contact person: } & \\ \text { first, last name: } & \text { Koen Martens } \\ \text { phone: } & +32(0) 26274315 \\ \text { email: } & \text { koen.martens@naturalsciences.be }\end{array}$


scientific contact person:
first, last name:
Koen Martens
phone:
+32(0)26274315
email:
koen.martens@naturalsciences.be

\title{
Intellectual property rights and citation
}

\author{
dataset creator (data compiler): \\ contact name: \\ Pieter Lemmens \\ contact email: \\ pieter.lemmens@kuleuven.be \\ contact institution: \\ KU Leuven
}

data contributors to/owners of this dataset:

multiple

number:

9

\section{data contributor/owner 1:}

contact name:

Koen Martens

contact email:

koen.martens@naturalsciences.be

contact institute:

RBINS

criteria for using this part of the dataset:

The dataset is publicly available (data portal, data archive) and can be used without restrictions, but dataset creator/data contributors must be informed prior to publication. Data must be acknowledged and cited correctly.

data contributor/owner 2:
contact name:
Wim Vijverman
contact email:
contact institute:
wim.vyverman@ugent.be
UGent

criteria for using this part of the dataset:

The dataset is publicly available (data portal, data archive) and can be used without restrictions, but dataset creator/data contributors must be informed prior to publication. Data must be acknowledged and cited correctly.

data contributor/owner 3:

contact name:

Patrick Kestemont

contact email:

patrick.kestemont@unamur.be

contact institute:

UNamur

criteria for using this part of the dataset:

The dataset is publicly available (data portal, data archive) and can be used without restrictions, but dataset creator/data contributors must be informed prior to publication. Data must be acknowledged and cited correctly.

data contributor/owner 4:

contact name:

contact email:

contact institute:
Leo Vanecke

leo.vanhecke@skynet.be

National Botanical Garden

criteria for using this part of the dataset:

The dataset is publicly available (data portal, data archive) and can be used without restrictions, but dataset creator/data contributors must be informed prior to publication. Data must be acknowledged and cited correctly.

\section{data contributor/owner 5:}

$\begin{array}{ll}\text { contact name: } & \text { Luc Denys } \\ \text { contact email: } & \text { luc.denys@inbo.be }\end{array}$


contact institute:

Institute for Nature and Forests

criteria for using this part of the dataset:

The dataset is publicly available (data portal, data archive) and can be used without restrictions, but dataset creator/data contributors must be informed prior to publication. Data must be acknowledged and cited correctly.

data contributor/owner 6:

contact name:

Marc Herremans

contact email:

contact institute:

marc.herremans@natuurpunt.be

criteria for using this part of the dataset:

The dataset is publicly available (data portal, data archive) and can be used without restrictions, but dataset creator/data contributors must be informed prior to publication. Data must be acknowledged and cited correctly.

\section{data contributor/owner 7:}

contact name:

contact email:

Luc De Meester

contact institute:

luc.demeester@kuleuven.be

criteria for using this part of the dataset:

The dataset is publicly available (data portal, data archive) and can be used without restrictions, but dataset creator/data contributors must be informed prior to publication. Data must be acknowledged and cited correctly.

data contributor/owner 8:

contact name:

Henry-Michel Cauchie

contact email:

henry-michel.cauchie@list.lu

contact institute:

CRP

criteria for using this part of the dataset:

The dataset is publicly available (data portal, data archive) and can be used without restrictions, but dataset creator/data contributors must be informed prior to publication. Data must be acknowledged and cited correctly.

\section{data contributor/owner 9:}

$\begin{array}{ll}\text { contact name: } & \text { Betrand Losson } \\ \text { contact email: } & \text { blosson@ulg.ac.be } \\ \text { contact institute: } & \text { ULiege }\end{array}$

criteria for using this part of the dataset:

The dataset is publicly available (data portal, data archive) and can be used without restrictions, but dataset creator/data contributors must be informed prior to publication. Data must be acknowledged and cited correctly.

citation of this dataset:

author(s):

Lemmens, P., Bonjean, N., Castiaux, A., Colson, L., De Bie, T., Decoster, E., Denis, C., De Roeck, E., Ercken, D., Goddeeris, B., Goyvaerts, R., Mandiki, S.N.M., Morelle, K., Praca, E., Schön, I., Van Wichelen, J., Van Der Gucht, K., Vandekerckhoven, J., Vanormelingen, P., Villena Alvarez, M., Bauwens, D., Denys, L., Herremans, M., Vanhecke, L., Losson, B., Caron, Y., Cauchie, H.-M., Kestemont, P., Vyverman, W., De Meester, L., Declerck, S.A.J. \& Martens, K. title and journal (name, number, pages):

Towards a sustainable management of pond diversity at the landscape level (PONDSCAPE)

year:

2018

doi:

https://doi.org/10.15468/zs6sky

citation of the metadata:

author(s):

Lemmens P., De Wever A., Bonjean N., Castiaux A., Colson L., De Bie T., 
Decoster E., Denis C., De Roeck E., Ercken D., Goddeeris B., Goyvaerts R., Mandiki S.N.M., Morelle K., Praca E., Schön I., Van Wichelen J., van der Gucht K., Vandekerckhoven J., Vanormelingen P., Villena Alvarez M., Bauwens D., Denys L., Herremans M., Vanhecke L., Losson B., Caron Y., Cauchie H.-M., Kestemont P., Vyverman W., De Meester L., Declerck S.A.J. \& Martens K. title and journal (name, number, pages):

Database of the PONDSCAPE project (Towards a sustainable management of pond diversity at the landscape level). Freshwater Metadata Journal 31: 1-10

year: 2018

doi:

https://doi.org/10.15504/fmj.2018.31

\section{dataset related references:}

reference 1:

author(s):

De Bie, T., De Meester, L., Brendonck, L., Martens, K., Goddeeris, B., Ercken, D., Hampel, H., Denys, L., Vanhecke, L., Van der Gucht, K., Van Wichelen, J., Vyverman, W., Declerck, S.A.

title:

Body size and dispersal mode as key traits determining metacommunity structure of aquatic organisms. Ecology Letters 15(7): 740-747.

year:

2012

\section{General data specifications}

regional coverage of the dataset: spatial extent of the dataset: national continents:

spatial extent (bounding coordinates):

southernmost latitude $\left[{ }^{\circ}\right]$ :

northernmost latitude $\left[^{\circ}\right]$ :

westernmost longitude $\left[{ }^{\circ}\right]$ :

2.3642

easternmost longitude $\left[{ }^{\circ}\right]$ :

6.5611

countries:

Europe: Belgium, Luxembourg

world climatic regions according to Köppen:

Group C: temperate/mesothermal climates

freshwater ecoregions of the world (FEOW) according to WWF:

Europe: Central \& Western Europe

\section{European ecoregions according to Illies (WFD):}

ecosystem type:

covered timeframe:

\section{Site specifications}

\section{coordinate system/grid data:}

datum (e.g. WGS84):

grid data available:

\section{site coding:}

site coding available:

number of digits:

example:

number of sites:
Western Plains (ER13)

lakes/ponds

$2008-2008$ 


\section{Climate and environmental data}

climate related data:

environmental data:

available parameters per site:

physico-chemical data: no climate data available

maximum depth

data source: as sampled

total $\mathrm{P}$, total $\mathrm{N}$, sulphate, chloride, calcium, hardness, alkalinity, oxygen content, water temperature, $\mathrm{pH}$, chlorophyll, Secci disc depth, suspended solids, sediment/soil parameters

availability of physico-chemical data, if there is more than one sample per site: mean values per site

stressors influencing the sites:

\section{Biological data}

biological data origin:

organism group addressed: from sampling, PONDSCAPE

amphibians, fish, macro-invertebrates (Mollusca, Coleoptera), zooplankton (Cladocera), phytoplankton, (benthic) diatoms, macrophytes, other group(s): bacterioplankton

\section{Sample specifications/sample resolution}

\section{amphibians: \\ sample information: \\ covered timeframe: \\ historical data: \\ season: \\ no \\ summer \\ temporal resolution/frequency of sampling: \\ time series data: \\ per year \\ no \\ species \\ level: \\ 100 \\ taxonomic coding:}

$2008-2008$ taxalist according to:

reference(s): see De Bie et al. (2012)

De Bie, T., De Meester, L., Brendonck, L., Martens, K., Goddeeris, B., Ercken, D., Hampel, H., Denys, L., Vanhecke, L., Van der Gucht, K., Van Wichelen, J., Vyverman, W., Declerck, S.A. (2012). Body size and dispersal mode as key traits determining metacommunity structure of aquatic organisms. Ecology Letters 15(7): $740-747$.

semi-quantitative 
replicate samples:

no

fish:

sample information:

covered timeframe:

$2008-2008$

historical data:

no

palaeo data:

no

season:

summer

temporal resolution/frequency of sampling:

time series data:

per year

no

taxonomic resolution:

level:

species

percentage of species level data: 100

\section{taxonomic coding:}

taxalist according to:

see De Bie et al. (2012)

reference(s):

De Bie, T., De Meester, L., Brendonck, L., Martens, K., Goddeeris, B., Ercken, D., Hampel, H., Denys, L., Vanhecke, L., Van der Gucht, K., Van Wichelen, J., Vyverman, W., Declerck, S.A. (2012). Body size and dispersal mode as key traits determining metacommunity structure of aquatic organisms. Ecology Letters 15(7): 740-747.

sample specifications:

type:

semi-quantitative

replicate samples:

no

\section{macro-invertebrates:}

sample information:

covered timeframe:

$2008-2008$

historical data:

no

palaeo data:

no

season:

summer

temporal resolution/frequency of sampling:

time series data:

per year

no

\section{taxonomic resolution:}

level:

percentage of species level data: 70

\section{taxonomic coding:}

taxalist according to: reference(s):

\section{sample specifications:}

type:

replicate samples:

\section{zooplankton:}

\section{sample information:}

covered timeframe:

historical data:

palaeo data: family, genus, species

70

see De Bie et al. (2012)

De Bie, T., De Meester, L., Brendonck, L., Martens, K., Goddeeris, B., Ercken, D., Hampel, H., Denys, L., Vanhecke, L., Van der Gucht, K., Van Wichelen, J., Vyverman, W., Declerck, S.A. (2012). Body size and dispersal mode as key traits determining metacommunity structure of aquatic organisms. Ecology Letters 15(7): 740-747.

semi-quantitative

no

$2008-2008$

no

no 
season: summer

temporal resolution/frequency of sampling:

time series data:

per year

taxonomic resolution:

level: $\quad$ species

percentage of species level data: 100

taxonomic coding:

taxalist according to:

see De Bie et al. (2012)

reference(s):

De Bie, T., De Meester, L., Brendonck, L., Martens, K., Goddeeris, B., Ercken, D., Hampel, H., Denys, L., Vanhecke, L., Van der Gucht, K., Van Wichelen, J., Vyverman, W., Declerck, S.A. (2012). Body size and dispersal mode as key traits determining metacommunity structure of aquatic organisms. Ecology Letters 15(7): 740-747.

sample specifications:

type:

semi-quantitative

replicate samples:

no

phytoplankton:

sample information:

covered timeframe:

$2008-2008$

historical data:

no

season:

summer

temporal resolution/frequency of sampling:

per year

time series data:

no

taxonomic resolution:

level:

genus

taxonomic coding:

taxalist according to:

reference(s):

see De Bie et al. (2012)

De Bie, T., De Meester, L., Brendonck, L., Martens, K., Goddeeris, B., Ercken, D., Hampel, H., Denys, L., Vanhecke, L., Van der Gucht, K., Van Wichelen, J., Vyverman, W., Declerck, S.A. (2012). Body size and dispersal mode as key traits determining metacommunity structure of aquatic organisms. Ecology Letters 15(7): 740-747.

sample specifications:

type:

replicate samples:

semi-quantitative

no

(benthic) diatoms:

sample information:

covered timeframe:

$2008-2008$

historical data:

no

palaeo data:

no

season:

summer

temporal resolution/frequency of sampling:

time series data:

per year

taxonomic resolution:

level:

no

percentage of species level data

family, genus, species

10

taxonomic coding:

taxalist according to:

see De Bie et al. (2012) 
reference(s):

sample specifications:

type:

replicate samples:

macrophytes:

sample information:

covered timeframe:

historical data:

palaeo data:

season:

temporal resolution/frequency of sampling:

time series data:

taxonomic resolution:

level:

percentage of species level data:

\section{taxonomic coding:}

taxalist according to:

reference(s):

no

no

no

no

species

100
De Bie, T., De Meester, L., Brendonck, L., Martens, K., Goddeeris, B., Ercken, D., Hampel, H., Denys, L., Vanhecke, L., Van der Gucht, K., Van Wichelen, J., Vyverman, W., Declerck, S.A. (2012). Body size and dispersal mode as key traits determining metacommunity structure of aquatic organisms. Ecology Letters 15(7): 740-747.

semi-quantitative

$2008-2008$

summer

per year

see De Bie et al. 2012

De Bie, T., De Meester, L., Brendonck, L., Martens, K., Goddeeris, B., Ercken, D., Hampel, H., Denys, L., Vanhecke, L., Van der Gucht, K., Van Wichelen, J., Vyverman, W., Declerck, S.A. (2012). Body size and dispersal mode as key traits determining metacommunity structure of aquatic organisms. Ecology Letters 15(7): 740-747.

sample specifications:

type:

quantitative (abundance data)

replicate samples:

no

other group(s):

sample information:

covered timeframe:

$2008-2008$

historical data:

no

season:

summer

temporal resolution/frequency of sampling:

per year

time series data:

no

\section{taxonomic resolution:}

level:

other taxonomic levels available

other taxonomic levels:

taxonomic coding:

taxalist according to:

sample specifications:

type:

DGGE bands

not relevant (DGGE bands)

semi-quantitative

replicate samples: 


\section{Other specifications}

GIS layers, shape files related to the dataset:

$\begin{array}{ll} & \text { no data available } \\ \text { availability of photos: } & \text { no } \\ \text { availability of maps: } & \text { no }\end{array}$

quality control procedures:

Were any quality control procedures applied to your dataset?

yes

quality control protocols and comments:

Species names were checked using the GBIF species list.

\section{Acknowledgements}

The authors acknowledge the Belgian Science Policy (Belspo) for funding the original PONDSCAPE project, as well as for funding the SAFRED project that allowed processing the Pondscape data.

\section{References}

De Bie, T., De Meester, L., Brendonck, L., Martens, K., Goddeeris, B., Ercken, D., Hampel, H., Denys, L., Vanhecke, L., Van der Gucht, K., Van Wichelen, J., Vyverman, W., Declerck, S.A., 2012. Body size and dispersal mode as key traits determining metacommunity structure of aquatic organisms. Ecology Letters 15(7): 740-747. 